\title{
Microbial Ecology and Antibiotic Susceptibility Profile of Germs Isolated from Hospital Surfaces and Medical Devices in a Reference Hospital in Douala (Cameroon)
}

\author{
Cécile Okalla Ebongue ${ }^{1,2,3^{*}}$, Chanceline Mayoudom Nguatcheussi², Jean-Pierre Nda Mefo'o1,2, \\ Joss Bollanga ${ }^{3}$, Dieudonné Adiogo ${ }^{2}$, Henry Namme Luma ${ }^{3}$ \\ ${ }^{1}$ Laboratory of Clinical Biology, General Hospital, Douala, Cameroon \\ ${ }^{2}$ Department of Biological Sciences, Faculty of Medicine and pharmaceutical Sciences, University of Douala, Douala, Cameroon \\ ${ }^{3}$ Committee for Hygiene and the Fight against Nosocomial Infections, General Hospital, Douala, Cameroon \\ Email: *cecileokalla@yahoo.fr, m.chan celynenina@yahoo.com,drnda41@yahoo.fr, bollangaj@yahoo.fr,d_adiogo@yahoo.fr, \\ hnluma@yahoo.com
}

How to cite this paper: Ebongue, C.O., Nguatcheussi, C.M., Mefo'o, J.-P.N., Bollanga, J., Adiogo, D. and Luma, H.N. (2018) Microbial Ecology and Antibiotic Susceptibility Profile of Germs Isolated from Hospital Surfaces and Medical Devices in a Reference Hospital in Douala (Cameroon). Advances in Microbiology, 8, 125-137. https://doi.org/10.4236/aim.2018.82009

Received: December 29, 2017 Accepted: February 25, 2018 Published: February 28, 2018

Copyright $\odot 2018$ by authors and Scientific Research Publishing Inc. This work is licensed under the Creative Commons Attribution International License (CC BY 4.0).

http://creativecommons.org/licenses/by/4.0/

\begin{abstract}
Background: The hospital environment is largely contaminated with pathogenic microorganisms. This colonization is a threat for hospitalized patients, especially in high-risk services. The purpose of this study was to identify the germs found on surfaces and medical devices in some departments of the General Hospital of Douala, and to establish their susceptibility profile to most commonly used antibiotics in this health facility. Results: We collected 114 surface and medical device samples, and seeded different culture media for Gram-positive and Gram-negative aerobic bacteria. Of the total samples, 108 were positive and 137 bacterial strains were isolated. The colony count revealed a high rate of contamination. Enterobacter cloacae was the most represented specie (53.3\%), followed by Pseudomonas aeruginosa (22.6\%) and Klebsiella pneumoniae (6.6\%). Various coagulase-negative Staphylococci have been isolated in some departments, as well as Cryptococcus laurentii and molds. The isolated strains showed low susceptibility to the antibiotics tested. Enterobacter cloacae showed low susceptibility for all tested molecules, except for carbapenems with rates ranging from $82 \%$ to over $94 \%$ in Maternity, Intensive Care and Neonatology units. The strains coming from the Haematology Protected Ward were resistant to all antibiotics, except fluoroquinolones with a susceptibility rate of $50 \%$ for ofloxacin. Conclusion: The hospital surfaces and medical devices are highly contaminated by environmental bacteria, with low susceptibility rates to antibiotics. Microbiological controls of the en-
\end{abstract}


vironment should be regular in critical areas in order to reinforce measures to prevent diffusion of multi-resistant bacteria.

\section{Keywords}

Microbial Ecology, Hospital Environment, Susceptibility to Antibiotics, Douala

\section{Introduction}

Microorganisms largely contaminate the hospital environment; this contamination is variable quantitatively and qualitatively from one institution to another and in a same establishment according to the services. It constitutes a risk factor for the occurrence of nosocomial infections, a real threat, both for the already precarious health of patients and that of the nursing staff and visitors. These infections increase health care costs and length of stay; and are the major cause of mortality and morbidity in hospitalized patients [1] [2]. Although it is difficult to establish a direct link between environmental contamination and the occurrence of nosocomial infections, several studies have shown that microorganisms of human and/or environmental origin contaminate hospital surfaces, and play an important role in the occurrence of these infections [3] [4]. The microorganisms involved in the hospital environment are most often multiresistant to antibiotics, and the main source of diffusion of highly pathogenic strains in services [5] [6] [7] [8]. Microbiological monitoring of the environment in health facilities is part of preventing the transmission of nosocomial infections. The microbiological controls of the environment are one of the measuring tools that make it possible to evaluate a starting situation and the effectiveness of corrective measures, they must be implemented in a relevant way and obey very precise objectives while avoiding the inflation of useless analyses, consuming time and financial means [9] [10] [11]. Efforts are being made to reduce contamination of the hospital environment by developing air and water monitoring methods, surfaces, food, medical devices, care equipment; and the strengthening of hospital hygiene measures [12]. The objective of this study was to quantify and qualify germs present on hospital surfaces and medical devices, and to study their antibiotic susceptibility profile.

\section{Material and Methods}

\subsection{Location and Type of Study}

We conducted a descriptive cross-sectional study at General Hospital of Douala (GHD) from $1^{\text {st }}$ January to $30^{\text {th }}$ June 2015 . This is a tertiary health facility located in the Littoral region of Cameroon. This hospital has a capacity of 320 beds and harbours all the major medical and surgical specialities. The choice of high-risk services and surfaces to be taken was made by convenience and focused on the 
medical-surgical Intensive Care Unit, the Operating Room, the Burn Unit, located at $1^{\text {st }}$ level on the right wing of the hospital; the Maternity and Neonatology ward located at the $2^{\text {nd }}$ level on the left wing; and the Protected Haematology Ward located at level 3 on the right wing. Microbiological analyses were carried out at the Bacteriology Unit of the Clinical Biology Laboratory at General Hospital of Douala.

\subsection{Sampling}

The most exposed surfaces, and low-traffic areas that often escape daily cleaning have been selected, as well as some medical devices. We excluded ceilings and walls in the samples. These samples were taken between $11 \mathrm{AM}$ and $01 \mathrm{PM}$ using a sterile swab previously moistened with sterile saline $0.9 \%$. The swabs were passed in parallel streaks by slightly turning them, on defined areas of $25 \mathrm{~cm}^{2}$.

\subsection{Seeding}

The swabs from the different sampling sites were eluted in the test tubes containing $2 \mathrm{ml}$ of sterile physiological saline to suspend the collected elements. The tubes were then vortexed for one minute, and 40 microliters of the resulting suspension were inoculated by the rake method into the following culture media: Plate Count Agar (PCA), Eosin Methylene Blue (EMB) Agar, Mannitol-Salt Agar, Sabouraud + Chloramphenicol (SC) Agar.

The dishes were then incubated in a bacteriological oven at $37^{\circ} \mathrm{C}$ for 24 hours for the bacteria, and 48 to 72 hours at $25^{\circ} \mathrm{C}$ for the fungi (SC Agar).

\subsection{Identification of Colonies}

Observation of petri dishes after incubation allowed for colony counting and macroscopic identification (shape, size colour). The biochemical and enzymatic identification of the microorganisms was made by seeding a suspension of microorganisms on cards; Vitek2 $\mathrm{GN}^{\mathrm{TM}}$ for Gram-negative fermenting and non-fermenting bacilli, Vitek2 $\mathrm{GP}^{\mathrm{TM}}$ for Gram-positive cocci and non-spore-forming bacilli, Vitek2 $\mathrm{YST}^{\mathrm{TM}}$ for yeast and yeast like organisms; followed by incubation and colorimetric reading on the VITEK2 Compact ${ }^{\mathrm{TM}} 15$, an automated microbiology system (bioMerieux SA, France).

\subsection{Susceptibility Tests}

They were performed using the diffusion technique of disks impregnated with antibiotics on Mueller Hinton medium incubated at $37^{\circ} \mathrm{C}$ for 24 hours. The following antibiotics discs corresponding to the most used molecules in the services, were studied based on the microorganisms isolated: Beta-lactamines (Amoxicillin + Clavulanic Acid and Third generation Cephalosporins), Carbapenems (Imipenem and Meropenem), Aminoglycosides (Gentamycin, Amikacin and Netilmicin), and Fluoroquinolones (Ofloxacin, Levofloxacin and Ciprofloxacin). The paper disks were applied, using a sterile plier, in $4 \mathrm{~mm}$ thickness Muel- 
ler-Hinton agar on $90 \mathrm{~mm}$ diameter Petri dishes, seeded with a bacterial suspension of $0.5 \mathrm{Mc}$ Farland. A $30 \mathrm{~mm}$ distance between the discs and the edge of the Petri dishes was respected in order to avoid overlapping zones of inhibition. After 24 hours incubation, the inhibition diameters around the antibiotic disk were measured, using a calliper. The values obtained permitted to classify the strains in three clinical categories: Sensitive (S), Intermediate (I) and Resistant (R), following the breakpoints of the committee of Susceptibility to antibiotics of the French Society of Microbiology (CASFM). In the same way, disks impregnated with antifungals (Amphotericin B, Nystatin, Fluconazole, Econazole and Ketoconazole) of determinated concentration were deposit on the surface of Sabouraud + Chloramphenicol medium, previously seeded with a calibrated inoculum prepared from the yeast culture to be tested. Inhibition diameter measurement and clinical categorisation were performed after $48 \mathrm{~h}$ incubation at $37^{\circ} \mathrm{C}$.

\subsection{Data Processing}

The results included the identification number, the date, the time and place of isolation, the identified germs, as well as the antibiotics tested and their susceptibility profile.

The descriptive analysis of the data was made using Epi Info ${ }^{\mathrm{TM}}$ version 7.0 and Microsoft Excel 2010 software.

\section{Results}

A total of 114 samples were collected from six at-risk and high-risk services, namely: Neonatology in the incubator room, the isolation room and the cloakroom (16); Maternity ward in the new-borns resuscitation room, the labour room and the delivery room (17); Medical and Surgical Intensive Care Unit (24); Burn Unit (23); Operating Room (21); and Haematology Protected Ward (13) (Table 1).

Of these 114 sampling sites, the majority were contaminated and only six (5.26\%) were uncontaminated, including the bench in the delivery room, 2 door handles and a neonatal incubator, a bubbler and equipment support in intensive care unit. In all other sectors, no uncontaminated area was noted.

The colony count gave a bacterial density $\geq 10^{6} \mathrm{CFU} / 25 \mathrm{~cm}^{2}$, except for the six samples with a sterile culture.

From the 108 contaminated specimens, we isolated 137 microorganisms, with a clear predominance of Enterobacter cloacae ssp cloacae (53.3\%), followed by Pseudomonas aeruginosa (22.6\%), Negative-coagulase Staphylococcus (8.7\%), and Klebsiella pneumoniae (6.6\%). For yeasts, 5 strains of Cryptococcus laurentii were isolated, and molds were present in two samples (Table 2).

E. cloacae is the only germ found in all services and on all types of supports (trolleys, beds, switches, door handles...), except for a sample taken from the bath of Burn Unit (Table 3). Pseudomonas aeruginosa was only found in two 
Table 1. Samples collected in different services.

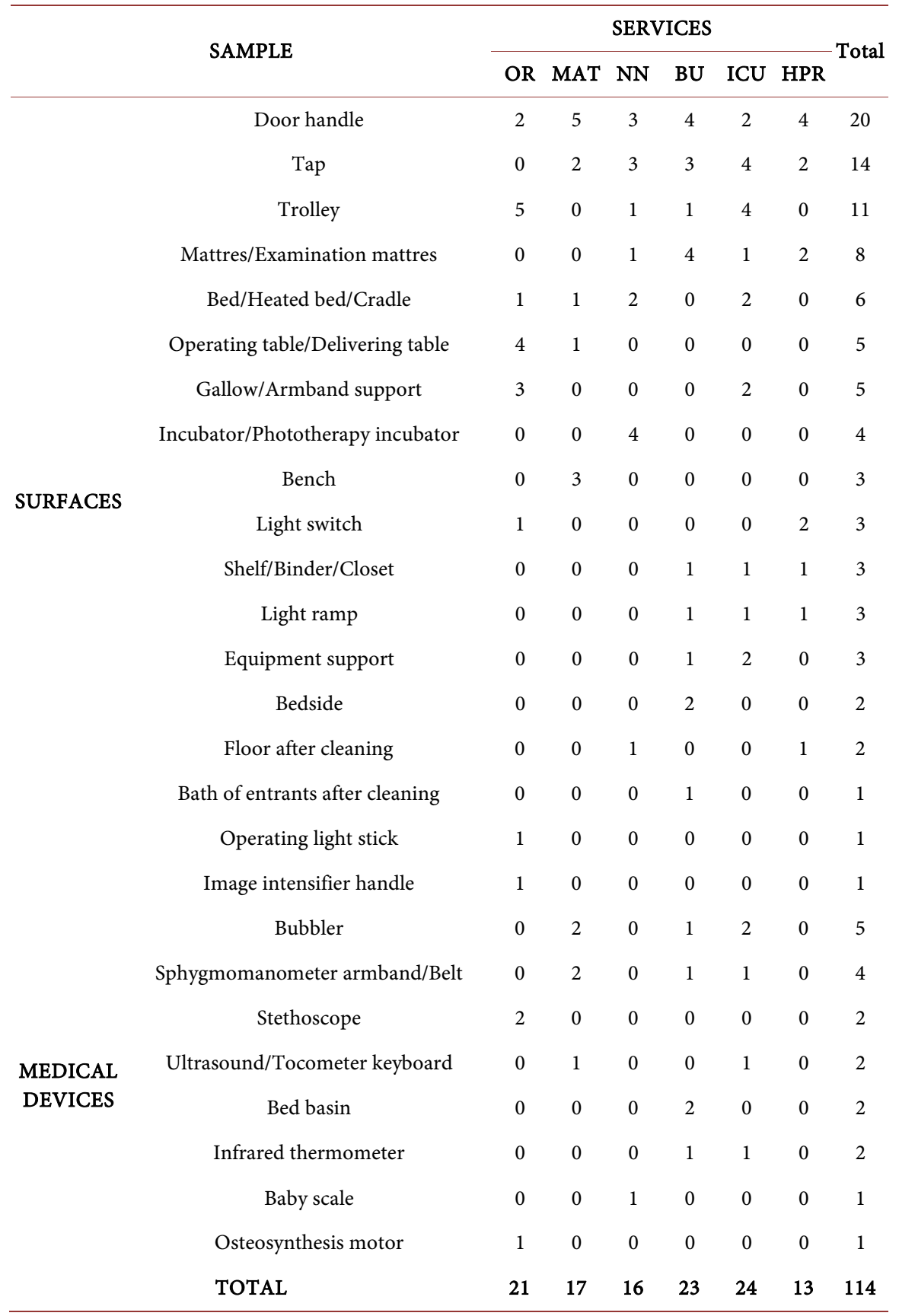

OR: operating Room; MAT: Maternity; NN: Neonatology; ICU: Intensive Care Unit; BU: Burn Unit; HPR: Hematology Protected Room.

departments, the Burn Unit and the Intensive Care Unit. As for Klebsiella pneumoniae, the strains were found in maternity, in neonatology, and in the Burn Unit (Table 2).

The study of the biochemical and enzymatic characteristics of the most observed strains showed a homology between the strains of Enterobacter cloacae isolated in the operating room and those of the intensive care unit, the burn unit, and the haematology protected ward. Similarly, strains isolated at maternity 
Table 2. Distribution of isolated microorganisms in the services.

\begin{tabular}{|c|c|c|c|c|c|c|c|c|}
\hline \multirow{2}{*}{\multicolumn{2}{|c|}{ Microorganisms }} & \multicolumn{7}{|c|}{ SERVICES } \\
\hline & & \multirow{2}{*}{$\frac{\text { OR }}{16}$} & \multirow{2}{*}{$\begin{array}{c}\text { MAT } \\
8\end{array}$} & \multirow{2}{*}{$\begin{array}{c}\text { NN } \\
7\end{array}$} & \multirow{2}{*}{$\begin{array}{c}\text { ICU } \\
17\end{array}$} & \multirow{2}{*}{$\begin{array}{l}\text { BU } \\
12\end{array}$} & \multirow{2}{*}{$\begin{array}{r}\text { HPR } \\
13\end{array}$} & \multirow{2}{*}{$\begin{array}{c}\text { TOTAL } \\
\text { n (\%) } \\
73(53.3)\end{array}$} \\
\hline & E. cloacae & & & & & & & \\
\hline Enterobacteriaceae & E. coli & 0 & 0 & 1 & 0 & 0 & 0 & $1(0.7)$ \\
\hline \multirow{5}{*}{$\begin{array}{c}\text { Gram Negative } \\
\text { Non Fermenting } \\
\text { Bacilli }\end{array}$} & $K \cdot$ pneumoniae & 0 & 2 & 3 & 0 & 4 & 0 & $9(6.6)$ \\
\hline & A. baumanii & 0 & 1 & 0 & 0 & 0 & 0 & $1(0.7)$ \\
\hline & A. salmonicida & 1 & 0 & 0 & 0 & 0 & 0 & $1(0.7)$ \\
\hline & B. cepacia & 0 & 1 & 0 & 0 & 1 & 0 & $2(1.5)$ \\
\hline & $P$. aeruginosa & 0 & 0 & 0 & 12 & 19 & 0 & $31(22.6)$ \\
\hline \multirow{7}{*}{$\begin{array}{c}\text { Negative } \\
\text { Coagulase } \\
\text { Staphylococcus }\end{array}$} & P. stuzzeri & 1 & 0 & 0 & 0 & 0 & 0 & $1(0.7)$ \\
\hline & S. carnosus & 0 & 1 & 0 & 0 & 0 & 0 & $1(0.7)$ \\
\hline & S. conhii & 1 & 0 & 0 & 1 & 0 & 0 & $2(1.5)$ \\
\hline & S. hominis & 0 & 1 & 0 & 0 & 0 & 0 & $1(0.7)$ \\
\hline & S. lentus & 1 & 0 & 0 & 0 & 0 & 0 & $1(0.7)$ \\
\hline & S. saprophyticus & 0 & 0 & 1 & 0 & 0 & 0 & $1(0.7)$ \\
\hline & S. sciuri & 0 & 0 & 0 & 0 & 1 & 0 & $1(0.7)$ \\
\hline \multirow{4}{*}{ Yeast and Molds } & S. warnei & 1 & 0 & 0 & 1 & 0 & 0 & $2(1.5)$ \\
\hline & S. xylosus & 0 & 0 & 0 & 1 & 1 & 0 & $2(1.5)$ \\
\hline & C. laurentii & 0 & 3 & 1 & 1 & 0 & 0 & $5(3.6)$ \\
\hline & Molds & 1 & 0 & 0 & 0 & 1 & 0 & $2(1.5)$ \\
\hline \multicolumn{2}{|c|}{ TOTAL n (\%) } & $\begin{array}{c}22 \\
(16.1)\end{array}$ & $\begin{array}{c}17 \\
(12.4)\end{array}$ & $\begin{array}{c}13 \\
(9.5)\end{array}$ & $\begin{array}{c}33 \\
(24.1)\end{array}$ & $\begin{array}{c}39 \\
(28.5)\end{array}$ & $\begin{array}{c}13 \\
(9.5)\end{array}$ & $\begin{array}{c}137 \\
(100)\end{array}$ \\
\hline \multicolumn{2}{|c|}{$P$ value } & 0.39 & 0.37 & 0.38 & 0.39 & 0.38 & 0.39 & \\
\hline
\end{tabular}

OR: Operating Room; MAT: Maternity; NN: Neonatology; ICU: Intensive Care Unit; BU: Burn Unit; HPR: Hematology Protected Room.

and those isolated in neonatology showed the same biochemical characteristics for Enterobacter cloacae and K. pneumonia (Table 4).

Regarding antimicrobial susceptibility, isolated strains showed low susceptibility levels. Enterobacter cloacae showed low susceptibility for all tested molecules, except for carbapenems with rates ranging from $82 \%$ to over $94 \%$ for isolated strains in Maternity, Intensive Care Unit and Neonatology. For the other services, the rates were lower than $37 \%$ for the same molecules (Figure 1). The strains coming from the Haematology Protected Ward were practically resistant to all antibiotics, the highest susceptibility rate being towards quinolones $(50 \%$ for ofloxacin) (Figure 1). The susceptibility of $K$. pneumoniae to the antibiotics tested is variable, ranging from $0 \%$ for amoxicillin-clavulanic acid in Maternity, to $100 \%$ for Carbapenems and Amikacin in Maternity and Neonatology (Figure 2). Pseudomonas aeruginosa strains showed low levels of susceptibility to the different molecules tested, especially in the Burn Unit where they vary from $0 \%$ for Quinolones, to $35 \%$ for Carbapenems. In the ICU, these rates were high 
Table 3. Microorganisms isolated from different supports.

\begin{tabular}{|c|c|c|c|c|c|c|c|c|c|}
\hline \multirow[b]{2}{*}{ SUPPORT } & \multicolumn{9}{|c|}{ MICROORGANISM } \\
\hline & E. cloacae & $\begin{array}{c}P . \\
\text { aeruginosa }\end{array}$ & $\begin{array}{c}K . \\
\text { pneumoniae }\end{array}$ & NCS & $\begin{array}{c}\text { Other } \\
\text { GNNFB }\end{array}$ & E. coli & $\begin{array}{c}C . \\
\text { laurentii }\end{array}$ & Molds & Total \\
\hline Door handle & 12 & 3 & 3 & 2 & 0 & 0 & 0 & 0 & 20 \\
\hline Tap & 8 & 5 & 2 & 0 & 0 & 1 & 4 & 0 & 20 \\
\hline Trolley & 8 & 4 & 1 & 1 & 0 & 0 & 0 & 0 & 14 \\
\hline Mattres & 6 & 5 & 0 & 0 & 0 & 0 & 0 & 0 & 11 \\
\hline Bed/Craddle & 4 & 0 & 0 & 2 & 0 & 0 & 1 & 0 & 7 \\
\hline Operating/Delivering table & 3 & 0 & 0 & 1 & 2 & 0 & 0 & 0 & 6 \\
\hline Gallow/armband support & 4 & 1 & 0 & 0 & 0 & 0 & 0 & 1 & 6 \\
\hline Incubator & 2 & 0 & 1 & 0 & 0 & 0 & 0 & 0 & 3 \\
\hline Bench & 1 & 0 & 0 & 0 & 0 & 0 & 0 & 0 & 1 \\
\hline Light switch & 3 & 0 & 0 & 0 & 0 & 0 & 0 & 0 & 3 \\
\hline Shelf/Binder/Closet & 2 & 1 & 0 & 0 & 0 & 0 & 0 & 0 & 3 \\
\hline Equipment support & 1 & 1 & 0 & 0 & 0 & 0 & 0 & 0 & 2 \\
\hline Light ramp & 2 & 2 & 0 & 0 & 0 & 0 & 0 & 0 & 4 \\
\hline Bedside & 2 & 2 & 0 & 0 & 0 & 0 & 0 & 0 & 4 \\
\hline Floor & 2 & 0 & 0 & 0 & 0 & 0 & 0 & 0 & 2 \\
\hline Bath & 0 & 0 & 0 & 1 & 1 & 0 & 0 & 0 & 2 \\
\hline Operating light & 1 & 0 & 0 & 0 & 0 & 0 & 0 & 0 & 1 \\
\hline Image intensifier & 1 & 0 & 0 & 0 & 0 & 0 & 0 & 0 & 1 \\
\hline Medical devices & 11 & 7 & 2 & 4 & 2 & 0 & 0 & 1 & 27 \\
\hline TOTAL & 73 & 31 & 9 & 11 & 5 & 1 & 5 & 2 & 137 \\
\hline$P$ value & 0.33 & 0.34 & 0.37 & 0.37 & 0.38 & 0.4 & 0.38 & 0.4 & \\
\hline
\end{tabular}

NCS: Negative-Coagulase Staphylococcus; GNNFB: Gram Negative Non Fermenting Bacilli.

for Carbapenems and Fluoroquinolones at $85 \%$ and $90 \%$, respectively (Figure 3). For Negative-Coagulase Staphylococcus, all the strains isolated in the Operating Room and in Neonatology were resistant to Oxacillin, and also $S$. carnosus and $S$. sciuri isolated in Maternity and Burn Unit, respectively. Concerning the susceptibility of $C$. laurentii to antifungal agents, the strains tested were sensitive to Fluconazole, Econazole and Ketoconazole, and resistant to Amphotericin B and Nystatin.

\section{Discussion}

The hospital environment is a real reservoir of microorganisms involved in many cases of nosocomial infections. Our study focused on the analysis of surface samples and medical devices in at-risk services in a reference hospital in Cameroon. The results of the culture showed that the surfaces and medical devices of the hospital were heavily contaminated by microorganisms. All samples taken at the Burn Unit, the operating theater and the protected hematology ward were positive. All these services are home to frail patients at very high risk of 
Table 4. Biochemical and enzymatic profile of E. cloacae, P. aeruginosa and K. pneumonia.

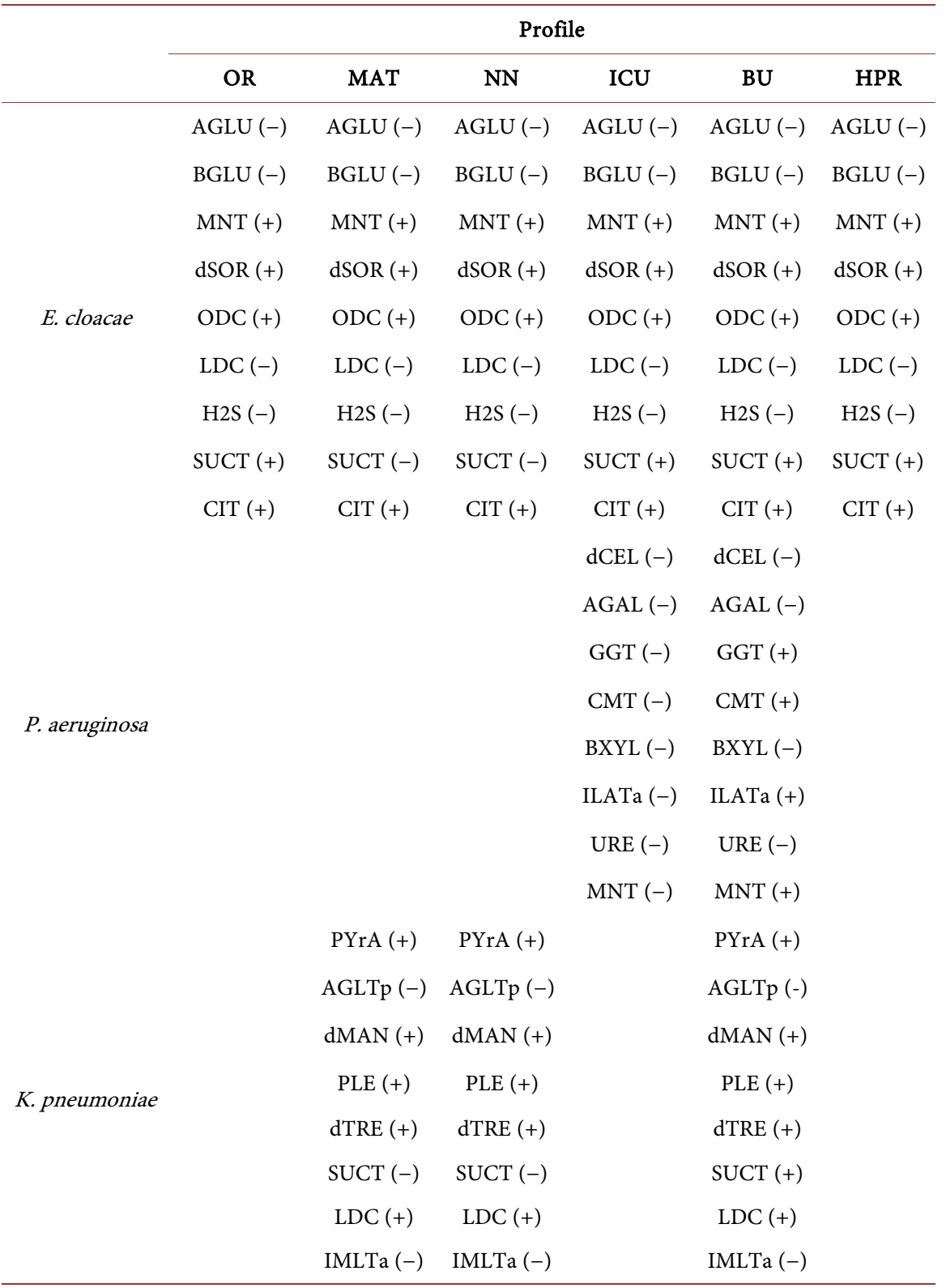

(+) Positive reaction; $(-)$ Negative reaction A; GLU = Alpha glucosidase; $\mathrm{BGLU}=$ Beta glucosidase; $\mathrm{MNT}=$ Malonate; $\mathrm{d}$ SOR $=\mathrm{D}$-Sorbitol; ODC $=$ Ornithine decarboxylase LDC $=$ Lysine decarboxylase; $\mathrm{H} 2 \mathrm{~S}=\mathrm{H} 2 \mathrm{~S}$ production; SUCT $=$ Succinate alkalinisation; CIT $=$ Sodium Citrate; $\mathrm{d}$ CEL $=$ D-Cellobiose; $A G A=$ Alpha galactosidase; GGT $=$ Gamma Glutamyl Transferase; $\mathrm{CMT}=$ Coumarate; $\mathrm{BXYL}=$ Beta xylosidase; ILA Ta $=$ L Lactate assimilation; URE $=$ Urease; PYrAL L $=$ Pyrrolodonyl-Arylamidase; AGLTp $=$ Glutamyl Arylamidase PNA; d MAN = D-Mannitol; PLE = Palatinose; $\mathrm{d}$ TRE $=$ D-Trehalose; $\mathrm{IMLTa}=\mathrm{L}$ Malate assimilation.

infection. Moreover, the high bacterial density on almost all samples regardless of the type of material, could result in a lack of cleaning or the use of non-effective antiseptics. De Abreu et al. showed variable contamination rates with the majority of highly contaminated media, but similar levels of contamination between intensive care, medicine and urology [13]. Non-critical medical devices such as stethoscopes are generally poorly contaminated, unlike those in 


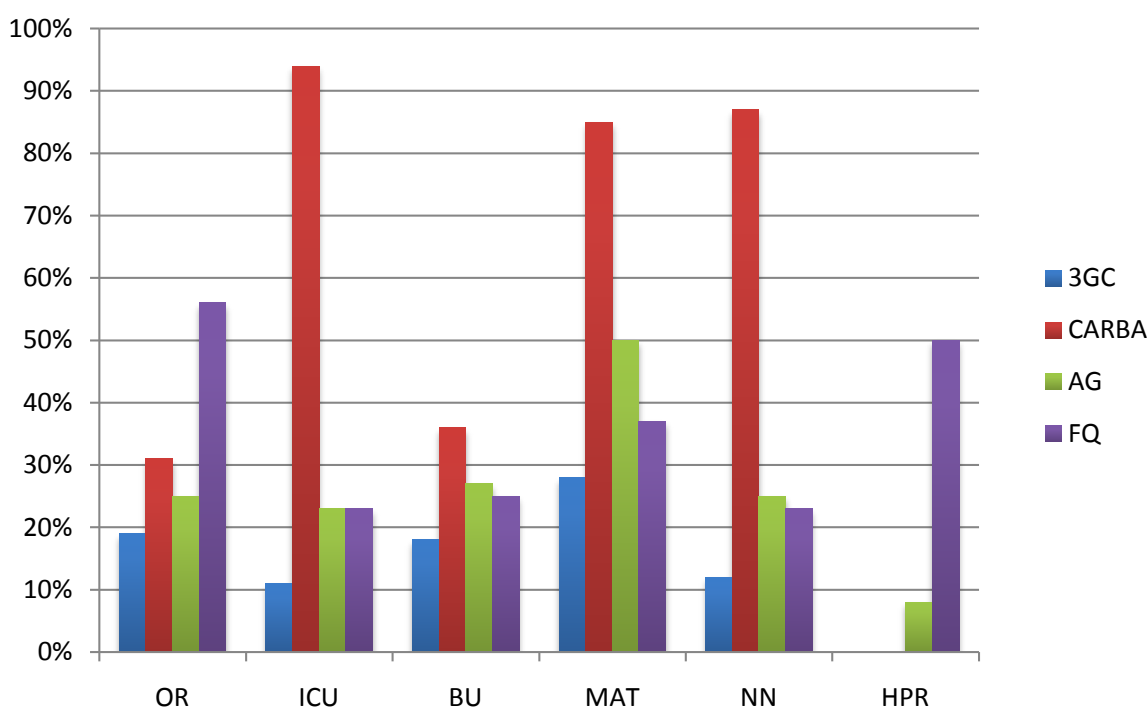

Figure 1. Susceptibility rates of Enterobacter cloacae. 3GC = Third generation Cephalosporin; CARBA = Carbapenems; $\mathrm{AG}=$ Aminoglycosides; $\mathrm{FQ}=$ Fluoroquinolones.

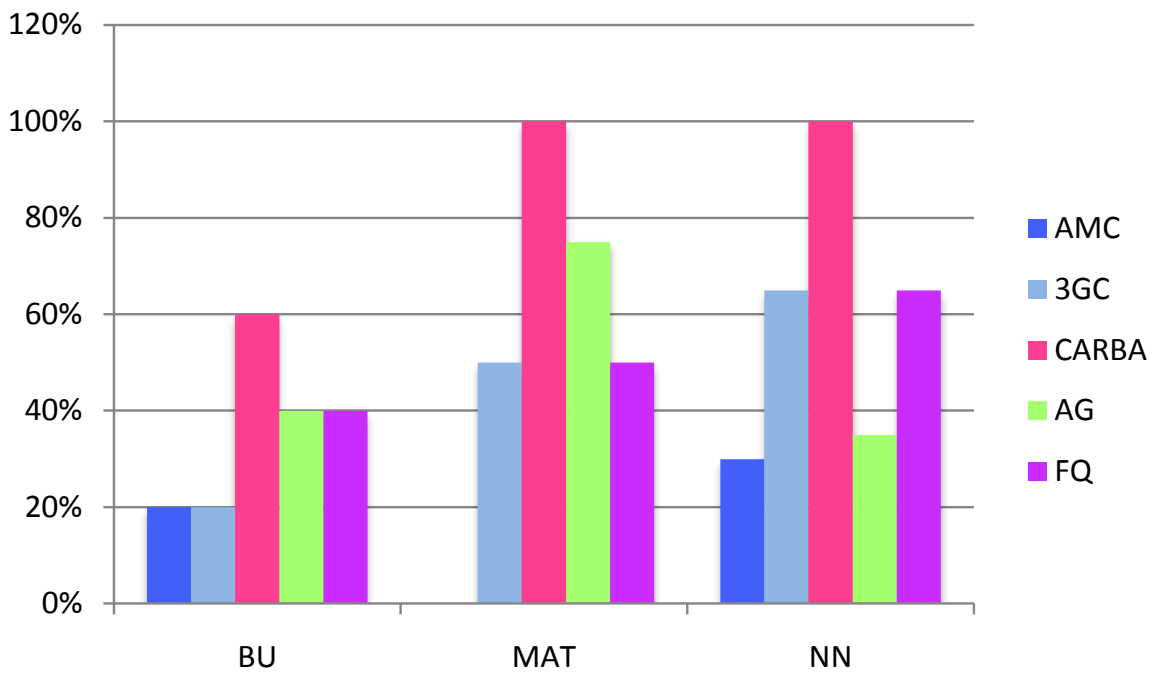

Figure 2. Susceptibility rates of Klebsiella pneumonia. AMC = Amoxicilline + Clavulanic Acid; $3 \mathrm{GC}=$ Third generation Cephalosporin; CARBA = Carbapenems; AG = Aminoglycosides; FQ = Fluoroquinolones.

our study, this may be due to the irregularity of the disinfection of these instruments. The swabbing method used may also play a role in the results of bacterial densities [11]. The high rate of contamination of these critical areas is paradoxical because they are protected areas, normally with low circulation of staff and visitors.

E. cloacae ssp cloacae is the most recovered species, although enterobacteria have little resistance to desiccation, hence their low presence in the environment [14]. The presence of $\mathrm{E}$ cloacae in all protected areas may be due to the transfer of patients from one Unit to another, the uncontrolled movement of staff, and lack of and hygiène, as demonstrated by Harbath et al. in a study done in a 


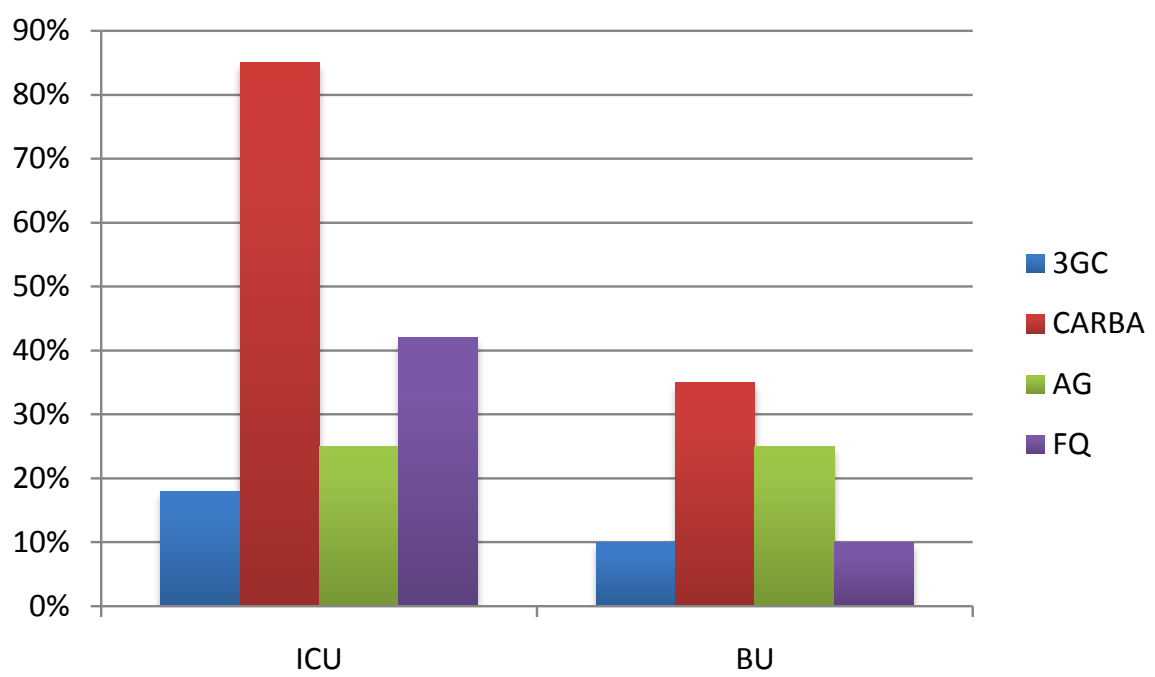

Figure 3. Susceptibility rates of Pseudomonas aeruginosa. 3GC $=$ Third generation Cephalosporin; CARBA = Carbapenems; $\mathrm{AG}=$ Aminoglycosides; $\mathrm{FQ}=$ Fluoroquinolones.

Neonatal Intensive Care Unit in Switzerland [15]. This transmission of E cloacae through patients transferred from one hospital to another has also been proven in some France hospitals that have received patients transferred from Morocco [16]. Some strains of $K$. pneumoniae have been isolated in Neonatalogy, Maternity and Burn Unit. Klebsiella pneumoniae may persist in the environment alone or in combination with Pseudomonas aeruginosa in mixed biofilms [17].

The $P$. aeruginosa were found in the Burn and Intensive Care Units, they are microorganisms for which humidity and temperature play an important role for their survival in the environment. Some authors have shown that Pseudomonas aeruginosa can survive from few months to several years on hospital surfaces [18]. Because of this ability to grow in wetlands, it is frequently found in the services of burn patients and Intensive Care, with varying susceptibility to antibiotics [19]. Isolated Pseudomonas in health facilities typically produce biofilms, and some genotypes are more productive than others. These biofilms are the starting point of the diffusion in services in taps and bedside tables; and can infect fragile patients such as burn victims [20]. Kominas et al. has demonstrated the transmission of germs, including Pseudomonas between health care staff and patients, and patients to patients in Intensive Care and Burn units [21].

For cocci, only coagulase-negative Staphylococci have been isolated, unlike other studies where $S$. aureus is predominant [14]. The presence of these SCN has been demonstrated on keyboards in permanent contact with the staff's bare fingers, while the use of gloves for other activities is systematic in these services [3]. Cryptococcus laurentii is the only yeast isolated in different services. This exclusivity may be due to the use of the Vitek $2^{\mathrm{TM}}$ automatic system for the identification of yeasts, because of the cross reactions between its capsular antigens and those of $C$. neoformans, identification errors are often observed as demonstrated by Xiao et al. [22]. The majority of Candida infections are of endogenous 
origin, however they can survive a few days in the hospital environment and be transmitted by the hands of caregivers and surfaces [14].

Although this is only a phenotypic identification, the biochemical homology between strains isolated in some services suggests transmission by staff and patients in the mother and child sector, and between the Operating Room and the Intensive Care Unit.

The susceptibility pattern shows that isolated strains have a low susceptibility rate for antibiotics commonly prescribed in services. Apart from Carbapenemes, which have good activity, resistance levels were high with respect to other molecules, particularly in the protected hematology ward. Chapuis et al. showed the role of the environment in the diffusion of broad-spectrum betalactamase-producing $E$. cloacae strains in a hematology service, both in the protected area and in the unprotected area [23].

\section{Limitations}

Limited ressources, as well as local technical platform did not allow us to perform molecular tests in order to type strains circulating in the hospital. In addition, since the GHD laboratory is specialized in clinical biology, we have not yet acquired new techniques for sampling and analyzing samples taken from the environment.

\section{Conclusion}

The investigated surfaces and medical devices of the Douala General Hospital were found to be highly contaminated by pathogenic environmental bacteria and certain yeasts; this allows us confirming the existence of a microbial ecology probably implicated in the occurrence of nosocomial infections. Isolated bacteria are weakly susceptible to the most commonly used antibiotics in these at-risk services. We recommend the reinforcement of staff, spaces and reusable medical devices hygiene, throughout the hospital and particularly in the high-risk areas. Microbiological controls of the environment should be regular in critical areas in order to reinforce measures to prevent diffusion of multi-resistant bacteria.

\section{Author's Contribution}

COE and DA drafted the manuscript; COE and HNL coordinated the study; CMN, JPNM and JB collected data and participated in its design. All the authors read and approved the final manuscript.

\section{Funding}

No funding was received in relation to this study.

\section{Ethics Approval}

This study was conducted in accordance with ethics directives related to research 
in Cameroon. We obtained the research authorization of the General Manager of the GHD, and ethical clearance from the institutional Ethics Committee of Research for Human Health from the University of Douala.

\section{References}

[1] Jarvis, W.R. (1996) Selected Aspects of the Socioeconomic Impact of Nosocomial Infections: Morbidity, Mortality, Cost, and Prevention. Infection Control \& Hospital Epidemiology, 17, 552-557. https://doi.org/10.2307/30141291

[2] Klevens, R.M., Edwards, J.R., Richards Jr., C.L., Horan, T.C., Gaynes, R.P., Pollock, D.A. and Cardo, D.M. (2007) Estimating Health Care-Associated Infections and Deaths in US Hospitals, 2002. Public Health Reports, 122, 160-166. https://doi.org/10.1177/003335490712200205

[3] Weber, D.J., Anderson, D. and Rutala, W.A. (2013) Current Opinion: The Role of the Surface Environment in Healthcare-Associated Infections. Current Opinion in Infectious Diseases, 26, 338-344. https://doi.org/10.1097/QCO.0b013e3283630f04

[4] Maki, D.G., Alvarado, C.J., Hassemer, C.A. and Zilz, M.A. (1982) Relation of the Inanimate Hospital Environment to Endemic Nosocomial Infection. New England Journal of Medicine, 307, 1562-1566. https://doi.org/10.1056/NEJM198212163072507

[5] Oie, S., Suenaga, S., Sawa, A. and Kamiya, A. (2007) Association between Isolation Sites of Methicillin-Resistant Staphylococcus Aureus (MRSA) in Patients with MRSA-Positive Body Sites and MRSA Contamination in Their Surrounding Environmental Surfaces. Japanese Journal of Infectious Diseases, 60, 367-369.

[6] Boyce, J.M. (2007) Environmental Contamination Makes an Important Contribution to Hospital Infection. Journal of Hospital Infection, 65, 50-54. https://doi.org/10.1016/S0195-6701(07)60015-2

[7] Wellington, E.M., Boxall, A.B., Cross, P., Feil, E.J., Gaze, W.H., Hawkey, P.M. and Thomas, C.M. (2013) The Role of the Natural Environment in the Emergence of Antibiotic Resistance in Gram-Negative Bacteria. The Lancet Infectious Diseases, 13, 155-65. https://doi.org/10.1016/S1473-3099(12)70317-1

[8] Talon, D. (1999) The Role of the Hospital Environment in the Epidemiology of Multi-Resistant Bacteria. Journal of Hospital Infection, 43, 13-17.

https://doi.org/10.1053/jhin.1999.0613

[9] Lemmen, S.W., Häfner, H., Zolldann, D., Amedick, G. and Lutticken, R. (2001) Comparison of Two Sampling Methods for the Detection of Gram-Positive and Gram-Negative Bacteria in the Environment: Moistened Swabs versus Rodac Plates. International Journal of Hygiene and Environmental Health, 203, 245-248. https://doi.org/10.1078/S1438-4639(04)70035-8

[10] el ayne Nabila, S., Adil, E., Abedelaziz, C., Nabila, A., Samir, H. and Abdelmajid, S. (2014) Rôle de l'environnement hospitalier dans la prévention des infections nosocomiales: Surveillance de la flore des surfaces à l'hôpital el Idrissi de Kenitra-Maroc. European Scientific Journal, 10.

[11] Mitchell, B.G., Wilson, F., Dancer, S.J. and McGregor, A. (2013) Methods to Evaluate Environmental Cleanliness in Healthcare Facilities. Healthcare Infection, 18, 23-30. https://doi.org/10.1071/HI12047

[12] Eckstein, B.C., Adams, D.A., Eckstein, E.C., Rao, A., Sethi, A.K., Yadavalli, G.K. and Donskey, C.J. (2007) Reduction of Clostridium difficile and Vancomycin-Resistant Enterococcus Contamination of Environmental Surfaces after an Intervention to 
Improve Cleaning Methods. BMC Infectious Diseases, 7, 61. https://doi.org/10.1186/1471-2334-7-61

[13] de Abreu, P.M., Farias, P.G., Paiva, G.S., Almeida, A.M. and Morais, P.V. (2014) Persistence of Microbial Communities Including Pseudomonas aeruginosa in a Hospital Environment: A Potential Health Hazard. BMC Microbiology, 14, 118. https://doi.org/10.1186/1471-2180-14-118

[14] Weinstein, R.A. and Hota, B. (2004) Contamination, Disinfection, and Cross-Colonization: Are Hospital Surfaces Reservoirs for Nosocomial Infection? Clinical Infectious Diseases, 39, 1182-1189. https://doi.org/10.1086/424667

[15] Harbarth, S., Sudre, P., Dharan, S., Cadenas, M. and Pittet, D. (1999) Outbreak of Enterobacter cloacae Related to Understaffing, Overcrowding, and Poor Hygiene Practices. Infection Control \& Hospital Epidemiology, 20, 598-603. https://doi.org/10.1086/501677

[16] Poirel, L., Ros, A., Carrër, A., Fortineau, N., Carricajo, A., Berthelot, P. and Nordmann, P. (2011) Cross-Border Transmission of OXA-48-Producing Enterobacter cloacae from Morocco to France. Journal of Antimicrobial Chemotherapy, 66, 1181-1182. https://doi.org/10.1093/jac/dkr023

[17] Vuotto, C., Longo, F., Balice, M.P., Donelli, G. and Varaldo, P.E. (2014) Antibiotic Resistance Related to Biofilm Formation in Klebsiella pneumoniae. Pathogens, 3, 743-758. https://doi.org/10.3390/pathogens3030743

[18] Chemaly, R.F., Simmons, S., Dale Jr, C., Ghantoji, S.S., Rodriguez, M., Gubb, J., Stibich, M., et al. (2014) The Role of the Healthcare Environment in the Spread of Multidrug-Resistant Organisms: Update on Current Best Practices for Containment. Therapeutic Advances in Infectious Disease, 2, 79-90. https://doi.org/10.1177/2049936114543287

[19] Davane, M., Suryawanshi, N., Pichare, A. and Nagoba, B.S. (2014) Pseudomonas aeruginosa from Hospital Environment. Journal of Microbiology and Infectious Diseases, 4, 42-43. https://doi.org/10.5799/ahinjs.02.2014.01.0124

[20] Vasiljević, Z., Jovčić, B., Ćirković, I. and Đukić, S. (2014) An Examination of Potential Differences in Biofilm Production among Different Genotypes of Pseudomonas aeruginosa. Archives of Biological Sciences, 66, 117-121. https://doi.org/10.2298/ABS1401117V

[21] Kominos, S.D., Copeland, C.E. and Grosiak, B. (1972) Mode of Transmission of Pseudomonas aeruginosa in a Burn Unit and an Intensive Care Unit in a General Hospital. Applied Microbiology, 23, 309-312.

[22] Xiao, M., Fan, X., Chen, X.X., Wang, H., Zhang, L., Xu, Z.P., Xu, Y.C., et al. (2016) Misidentification of a Rare Species, Cryptococcus laurentii, by Commonly Used Commercial Biochemical Methods and Matrix-Assisted Laser Desorption Ionization-Time of Flight Mass Spectrometry Systems: Challenges for Clinical Mycology Laboratories. Journal of Clinical Microbiology, 54, 226-229.

https://doi.org/10.1128/JCM.02830-15

[23] Chapuis, A., Amoureux, L., Bador, J., Gavalas, A., Siebor, E., Chrétien, M.L., Neuwirth, C., et al. (2016) Outbreak of Extended-Spectrum Beta-Lactamase Producing Enterobacter cloacae with High MICs of Quaternary Ammonium Compounds in a Hematology Ward Associated with Contaminated Sinks. Frontiers in Microbiology, 7, 1070. https://doi.org/10.3389/fmicb.2016.01070 